\title{
Qualitätssicherung und -entwicklung ja - aber mit welchen Instrumenten?
}

\author{
Projektteam: \\ Varja Meyer ${ }^{a}$, \\ Hélène Beutler ${ }^{b}$, \\ Johannes Brühwilerc, \\ Christoph Gehrlach ${ }^{d}$, \\ Adrian Rohrbassere \\ a $\mathrm{FMH}$, Schweizerische \\ Akademie für Qualität in \\ der Medizin SAQM (Projekt- \\ leitung) \\ b Verbindung der psychiatrisch- \\ psychotherapeutisch tätigen \\ Ärztinnen und Ärzte der \\ Schweiz FMPP \\ c Hausärzte Schweiz MFE \\ d Kompetenzzentrum \\ Qualitätsmanagement, \\ Berner Fachhochschule \\ e Schweizerische Gesellschaft \\ für Allgemeinmedizin SGAM
}

Verschiedene Erhebungen der Schweizerischen Akademie für Qualität in der Medizin SAQM der FMH zeigen auf, dass die Bandbreite von Qualitätsaktivitäten enorm gross ist. Qualitätsaktivitäten oder -instrumente wie beispielsweise interdisziplinäre Fallbesprechungen, Qualitätszirkel, Patientenbefragungen usw. gehören zum Alltag von Ärztinnen und Ärzten. Was diese Aktivitäten aber im Einzelnen und im jeweiligen Kontext tatsächlich an Nutzen und an Aufwand bedeuten, ist bis heute in den meisten Fällen nicht ermittelt. Um jedoch einen fundierten und breit durchdachten Entscheid für oder gegen die Einführung eines Instrumentes fällen zu können, braucht es eine systematische Be- und Durchleuchtung einzelner Instrumente hinsichtlich ihres Nutzens bei Implementierung und dem Aufwand bei Einführung und im Routinebetrieb. Daher hat die SAQM sich mit einem interdisziplinären Projektteam das Ziel gesetzt, ein praxisnahes, strukturiertes Bewertungsinstrument aus der Praxis für die Praxis litätsaktivitäten zu analysieren. Der Erfolg des Tools ist am grössten, wenn es mittels einer professionell moderierten Gruppendiskussion durchgeführt wird. Idealerweise setzt sich die Gruppe aus 4-8 Fachpersonen zusammen, die mit dem Thema Qualität im Allgemeinen und mit den zu bewertenden Qualitätsaktivitäten im Speziellen vertraut sind.

\section{Ablauf der Aufwand-Nutzen-Analyse}

Vor der eigentlichen Bewertung bestimmt die interessierte Gruppe, welche Qualitätsaktivität sie bewerten möchte. Der anschliessende Bewertungsprozess findet zusammen mit einem professionellen und mit dem Instrument vertrauten Moderator im Rahmen einer Einzelbewertung und nachfolgender strukturierter Gruppendiskussion statt. Bewertet werden verschiedenste Nutzen- und Aufwandaspekte jeweils betreffend die Patientinnen/Patienten, die Ärztinnen/Ärzte sowie die Arztpraxis.

\section{Das erarbeitete Analyseverfahren steht interessierten Organisationen und Personen gratis zur Verfügung.}

zu entwickeln. Genauere Angaben zu den Hintergründen der Entwicklungsarbeiten sowie ein detaillierter Überblick über das Bewertungsvorgehen wurden vor einem guten Jahr in der Schweizerischen Ärztezeitung publiziert [1]. Unterdessen wurde das Bewertungstool «Aufwand-Nutzen-Analyse von Qualitätsaktivitäten AQA» von verschiedenen Fachgruppen in der Praxis getestet. Aufgrund der positiven Ergebnisse aus dem Praxistest stellt die SAQM das erarbeitete Analyseverfahren nun interessierten Organisationen und Personen gratis zur Verfügung.

Korrespondenz:

FMH / SAQM, Abteilung DDQ

Elfenstrasse 18

CH-3000 Bern 15

Tel. 0313591111

Fax 0313591112

saqm[at]fmh.ch

\section{Zielgruppe}

Die entwickelte Aufwand-Nutzen-Analyse von Qualitätsaktivitäten richtet sich an verschiedene Gruppen wie beispielsweise Fachgesellschaften, Gruppenpraxen, Ärztenetzwerke oder Qualitätszirkel, die daran interessiert sind, einzelne oder mehrere Qua-

\section{Kontakt}

Sind Sie an einer Bewertung von Qualitätsaktivitäten interessiert? Mehr Informationen zur Aufwand-Nutzen-Analyse von Qualitätsaktivitäten finden Sie im Internet unter: www.saqm.ch $\rightarrow$ Qualitätsprojekte $\rightarrow$ Aufwand-Nutzen-Analyse von Qualitätsaktivitäten AQA. Die weiteren Unterlagen wie das Bewertungsraster, die Anleitung für Moderatorinnen und Moderatoren, eine Präsentation zur Einführung ins Analyseinstrument sowie ein Glossar geben wir Ihnen auf Anfrage gerne weiter. Diesbezüglich sowie bei weiteren Fragen steht Ihnen die Projektleiterin Varja A. Meyer gerne zur Verfügung (saqm[at]fmh. ch oder Telefon 03135911 11).

1 Beutler H, Brühwiler J, Gehrlach C, Hersperger M, Kurz R, Meyer V, Rohrbasser A. Nutzen und Aufwand von Qualitätsaktivitäten: ein neues Tool bringt Licht ins Dunkel. Schweiz Ärztezeitung. 2013;94(22):817-8. 